J. Bangladesh Acad. Sci., Vol. 42, No. 2, 191-199, 2018

DOI: https://doi.org/10.3329/jbas.v42i2.40051

\title{
ON A RECURRENCE RELATION RELATED TO THE REVE'S PUZZLE
}

\author{
A. A. K. MAJUMDAR
}

Ritsumeikan Asia-Pacific University, 1-1 Jumonjibaru, Beppu 874-8577, Japan

\section{ABSTRACT}

The 4-peg Tower of Hanoi problem, commonly known as the Reve's puzzle, is well-known. Motivated by the optimality equation satisfied by the optimal value function M(n) satisfied in case of the Reve's puzzle, (Matsuura et al. 2008) posed the following generalized recurrence relation

$$
T(n, \alpha)=\min _{1 \leq t \leq n}\{\alpha T(n-t, \alpha)+S(t, 3)\},
$$

where $\mathrm{n} \geq 1$ and $\alpha \geq 2$ are integers, and $S(t, 3)=2^{t}-1$ is the solution of the 3 -peg Tower of Hanoi problem with t discs. Some local-value relationships are satisfied by $T(n, \alpha)$ (Majumdar et al. 2016). This paper studies the properties of $T(n+1, \alpha)-T(n, \alpha)$ more closely for the case when $\alpha$ is an integer not of the form $2^{i}$ for any integer $i \geq 2$.

Keywords: Reve's puzzle, recurrence relation, local-value relationships

\section{INTRODUCTION}

The Reve's puzzle is a generalization of the Tower of Hanoi problem, where there are four pegs (instead of three). Briefly, the problem is as follows : There are four pegs and $n(\geq 1)$ discs of different sizes. Initially, the discs rest on one of the four pegs in a tower in small-on-large ordering. The problem is to shift the tower to another peg in minimum number of moves, where each move can transfer only one (topmost) disc from one peg to another such that no disc is ever placed on top of a smaller one. Denoting by $M(n)$ the minimum number of moves required to solve the Reve's puzzle, M(n) satisfies the dynamic programming equation

$$
M(n)=\min _{0 \leq k \leq n-1}\{2 M(k)+S(n-k, 3)\} ; n \geq 1,
$$

where $S(k, 3)=2^{k}-1$ is the number of moves required to solve the 3-peg Tower of Hanoi problem with $\mathrm{k} \geq 1$ number of discs. For details on the Reve's puzzle, one can consult Roth et al.
(1974); Hinz et al. (1989); Stockmeyer et al. (1994) and Majumdar et al. (1994 \& 2012).

Motivated by the above recurrence relation for M(n), Matsuura et al. (2008) introduced the following recurrence relation

$T(n, \alpha)=\min _{0 \leq k \leq n-1}\{\alpha T(k, \alpha)+S(n-k, 3)\} ; n \geq 1$,

which is equivalent to the one below :

$T(n, \alpha)=\min _{1 \leq t \leq n}\{\alpha T(n-t, \alpha)+S(t, 3)\} ; n \geq 1$,

$T(0, \alpha)=0$,

where $\alpha \geq 2$ is an integer.

In a recent paper, Matsuura et al. (2016) studied the above recurrence relation, and derived some new results, including some local-value relationships satisfied by the optimal value function $T(n, \alpha)$. The main results are reproduced below for reference later.

Lemma 1.1 : $T(n+1, \alpha)-T(n, \alpha) \geq 2$ for all $\mathrm{n} \geq 1, \alpha \geq 3$.

* Corresponding author: <aakmajumdar@gmail.com>. Ritsumeikan Asia-Pacific University, 1-1 Jumonjibaru, Beppu 874-8577, Japan 
Lemma 1.2 : For any $\alpha \geq 3$ fixed, for all $n \geq 1$,

$T(n+1, \alpha)-T(n, \alpha) \leq T(n+2, \alpha)-T(n+1, \alpha) \leq$ $2[T(n+1, \alpha)-T(n, \alpha)]$.

Lemma 1.3 : For any $\alpha \geq 3$ fixed, let $T(n, \alpha)$ be attained at the point $\mathrm{k}=\mathrm{k}_{1}$ and $\mathrm{T}(\mathrm{n}+1$, $\alpha)$ be attained at the point $k=k_{2}$. Then, $k_{1} \leq k_{2} \leq k_{1}+1$.

In Lemma 1.3 above, if $T(n, \alpha)$ is attained at more than one value of $k$, then $k_{1}$ is the minimum of such values of $\mathrm{k}$, and $\mathrm{k}_{2}$ is the minimum of the values of $\mathrm{k}$ at which $T(n+1, \alpha)$ is attained. Alternatively, $\mathrm{k}_{1}$ may be taken as the maximum of the values of $\mathrm{k}$ at which $T(n, \alpha)$ is attained, and in such a case, $\mathrm{k}_{2}$ is the maximum of the values of $\mathrm{k}$ at which $T(n+1, \alpha)$ is attained.

Lemma 1.4: If $\alpha \neq 2^{i}$ (for any integer $i \geq 2$ ), then $T(n, \alpha)$ is attained at a unique $\mathrm{k}$.

It may be mentioned here that, if $\alpha=2^{\mathrm{i}}$ for some integer $i \geq 1$, then the result of Lemma 1.4 does not hold true. It may be recalled that, for the Reve's puzzle, the corresponding optimal value function $M(n)$ is attained at exactly two values of $\mathrm{k}$ if and only if $\mathrm{n}$ is a triangular number; in other cases, $M(n)$ is attained at a unique k. Again, though each of $T(1,4)$ and $T(2,4)$ is attained at a unique point, $T(3,4)$ is attained at two values of $\mathrm{k}$, namely, at $\mathrm{k}=0,1$.

Lemma 1.5 : For any $\alpha \geq 3$ fixed, let $T(n, \alpha)$ and $T(n+1, \alpha)$ both be attained at $k=K$. Then,

$T(n+1, \alpha)-T(n, \alpha)=2^{n-K}$.

Moreover, if $\alpha$ is an integer not of the form $2^{i}$ for any integer $\mathrm{i} \geq 2$, then neither $T(n-1, \alpha)$ nor $T(n+2, \alpha)$ is attained at $\mathrm{k}=\mathrm{K}$.

Lemma 1.6 : Let $\alpha \neq 2^{i}$ for any integer $\mathrm{i} \geq 2$. Then

$T(n+1, \alpha)-T(n, \alpha)=2^{s}$ (for some integer $\left.s \geq 1\right)$ if and only if $T(n+1, \alpha)$ and $T(n, \alpha)$ both are attained at the same $\mathrm{k}$.
Lemma 1.6 differentiates between the cases when $\alpha=2^{i}$ (for any integer $\mathrm{i} \geq 1$ ) or not. It may be noted that, for all $\mathrm{n} \geq 1, M(n+1)-M(n)=2^{s}$ for some integer $s \geq 1$. In fact, it can be shown that, if $\alpha=2^{i}$ (for any integer $i \geq 1$ ), then for all $n \geq$ $1, T(n+1, \alpha)-T(n, \alpha)=2^{s}$ for some integer $\mathrm{s} \geq 1$.

Lemma 1.7 : For any $\mathrm{n} \geq 3$ and $\alpha \geq 3$ fixed, $T_{\alpha}(n, k)$ is convex in $\mathrm{k}$ in the sense that $T_{\alpha}(n, k+2)-T_{\alpha}(n, k+1) \geq T_{\alpha}(n, k+1)-T_{\alpha}(n, k)$ for all $0 \leq k \leq n-3$.

where $T_{\alpha}(n, k)=\alpha T(k, \alpha)+S(n-k, 3)$.

Corollary 1.1: $T(n+1, \alpha)$ is attained at $\mathrm{k}=0$ (for some integers $\mathrm{n}$ and $\alpha$ ) if and only if $\alpha>2^{n}$.

It is also noted that, the case $\alpha=2$ corresponds to the Reve's puzzle, which has been studied in detail Matsuura et al. (1994). Also, that some of the results above are valid for $\alpha=2$ as well. More specifically, each of Lemma 1.1, Lemma 1.2, Lemma 1.3 and Corollary 1.1 holds true when $\alpha=$ 2. This paper gives some local-value relationships involving $T(n+1, \alpha)-T(n, \alpha)$, when $\alpha \neq 2^{i}$ for any integer $\mathrm{i} \geq 2$. They are given in Section 2. These local-value relationships reveal the combinatorial properties inherent in the recurrence relation (1.2). In Section 3, the main result of the paper is estalished. Let $a_{n}=T(n, \alpha)-T(n-1, \alpha), n \geq 1$.

Let $\left\{b_{n}\right\}_{n=1}^{\infty}$ be the sequence of integers $b_{n}=2^{i} \alpha^{m} ; i \geq 0, m \geq 0$,

arranged in (strictly) increasing order. It can be proved that $a_{n}=b_{n}$ by showing that they satisfy the same recursion formulas. This shows that the recurrence relation satisfied by the sequence of numbers $\left\{a_{n}\right\}_{n=1}^{\infty}$ can be derived independently, without making use of the properties of sequence $\left\{b_{n}\right\}_{n=1}^{\infty}$. The paper can be considered as concluded, however with some remarks in section 4 . 


\section{PRELIMINARY RESULTS}

In this section, some preliminary results are derived that would be required in proving the main result in Section 3. Some local-value relationships satisfied by $T(n, \alpha)$, when $\alpha \geq 3$ is an integer, not of the form $2^{i}$ for any integer $i \geq 2$ are also solved below.

Lemma 2.1: Let $2^{i}<\alpha<2^{i+1}$ for some integer $i \geq 1$. Then,

(a) $T(i+1, \alpha)$ is attained at $\mathrm{k}=0$,

(b) each of $T(i+2, \alpha)$ and $T(i+3, \alpha)$ is attained at $\mathrm{k}=1$,

(c) $T(i+4, \alpha)$ is attained at $\mathrm{k}=2$.

Proof : Part (a) follows from Corollary 1.1.

(b) The first to note is:

$T_{\alpha}(n, 1) \equiv \alpha T(1, \alpha)+S(n-1,3)<S(n, 3) \equiv T_{\alpha}(n, 0)$

if and only if $\alpha<2^{n-1}$, and

$T_{\alpha}(n, 2) \equiv \alpha T(2, \alpha)+S(n-2,3)>\alpha T(1, \alpha)+S(n-$ $1,3) \equiv T_{\alpha}(n, 1)$

if and only if $\alpha>2^{n-3}$.

Thus, by Corollary $1.1, T(n, \alpha)$ is attained at $\mathrm{k}=1$ if and only if $2^{n-3}<\alpha<2^{n-1}$.

In particular, $T(i+2, \alpha)$ and $T(i+3, \alpha)$ both are attained at $\mathrm{k}=1$.

(c) $T(i+4, \alpha)$ is not attained at $\mathrm{k}=1$, for otherwise

$T(i+4, \alpha)=\alpha T(1, \alpha)+2^{i+3}-1<\alpha T(2, \alpha)+2^{i+2}-1$, so that $\alpha>2^{i+1}$. But this inequality contradicts the assumption.

Corollary 2.1 : Let $2^{i}<\alpha<2^{i+1}$ for some integer $\mathrm{i} \geq 2$. Then, each of $T(i-1, \alpha)$ and $T(i, \alpha)$ is attained at $\mathrm{k}=0$.

Proof : From part (a) of Lemma 2.2, together with Lemma 1.3, it is seen that $T(i, \alpha)$ is attained at $\mathrm{k}=0$. This, in turn, proves that $T(i-1, \alpha)$ is attained at $\mathrm{k}=0$.
Corollary 2.1 above is valid for $\mathrm{i}=1$ as well if the convention that $T(0, \alpha)$ is attained at the point $\mathrm{k}=0$.

Lemma 2.2 : Let $2^{\mathrm{i}}<\alpha<2^{\mathrm{i}+1}$ for some integer $\mathrm{i}$ $\geq 1$. Let, for some integer $\mathrm{n}(\geq 1)$, both $T(n, \alpha)$ and $T(n+1, \alpha)$ be attained at the point $\mathrm{k}=\mathrm{i}$. Then, $T(n+2, \alpha)$ is attained at the point $\mathrm{k}=\mathrm{i}+1$.

Proof : Let $T(n, \alpha)$ and $T(n+1, \alpha)$ both be attained at $\mathrm{k}=\mathrm{i}$. Then, by Lemma $1.5, T(n+2, \alpha)$ is not attained at $\mathrm{k}=\mathrm{i}$. Hence, by Lemma 1.3, $T(n+2, \alpha)$ must be attained at $k=i+1$, which was intended to be proved.

Lemma 2.3 : Let $2^{i}<\alpha<2^{i+1}$ for some integer i $\geq 1$. Let, for some integer $\mathrm{n} \geq 1, T(n, \alpha)$ be attained at $k=i-1$ and $T(n+1, \alpha)$ be attained at $\mathrm{k}=\mathrm{i}$. Then, $T(n+2, \alpha)$ is not attained at $k=i+1$.

Proof: Since $T(n, \alpha)$ is attained $\mathrm{k}=\mathrm{i}-1$,

$T(n, \alpha)=\alpha T(i-1, \alpha)+2^{n-i+1}-1<\alpha T(i, \alpha)+2^{n-i}-1$, which gives

$\alpha[T(i, \alpha)-T(i-1, \alpha)]>2^{n-i}$.

Now, by Corollary 2.1,

$$
T(i, \alpha)-T(i-1, \alpha)=2^{i-1},
$$

and hence, $\alpha>2^{n-2 i+1}$.

Again, since

$T(n+1, \alpha)=\alpha T(i, \alpha)+2^{n-i+1}-1<\alpha T(i-1$, $\alpha)+2^{n-i+2}-1$,

we get

$\alpha[T(i, \alpha)-T(i-1, \alpha)]<2^{n-i}$

so that,

$\alpha<2^{n-2 i+2}$.

Then, $T(n+2, \alpha)$ is not attained at $k=i+1$, for otherwise,

$T(n+2, \alpha)=\alpha T(i+1, \alpha)+2^{n-i+1}-1<\alpha T(i, \alpha)+2^{n-i+2}-1$, so that, $\alpha[T(i+1, \alpha)-T(i, \alpha)]<2^{n-i}$.

Now, by Lemma 2.1 and Corollary 2.1, $T(i+1$, $\alpha)$ and $T(i, \alpha)$ both are attained at the point $\mathrm{k}=0$. Therefore,

$T(i+1, \alpha)-T(i, \alpha)=2^{i}$.

Thus, $\alpha<2^{n-2 i+1}$, 
and a contradiction is reached. Hence, $T(n+2, \alpha)$ is not attained at $\mathrm{k}=\mathrm{i}+1$, and consequently, by Lemma $1.3, T(n+2, \alpha)$ is attained at the point $\mathrm{k}=\mathrm{i}$.

Let $2^{i}<\alpha<2^{i+1}$ for some integer $\mathrm{i} \geq 1$. Then, by Lemma 2.1 and Corollary 2.1, $T(i-1, \alpha), T(i, \alpha)$ and $T(i+1, \alpha)$ each is attained at the point $\mathrm{k}=0$. When $\alpha=3$, both $\mathrm{T}(1,3)$ and $\mathrm{T}(2,3)$ are attained at the point $\mathrm{k}=0$ (and by Lemma 2.2, $\mathrm{T}(3,3)$ and $\mathrm{T}(4,3)$ are attained at the point $\mathrm{k}=1)$, for $5 \leq \alpha \leq$ 7 , each of $\mathrm{T}(1, \alpha), \mathrm{T}(2, \alpha)$ and $\mathrm{T}(3, \alpha)$ is attained at the point $\mathrm{k}=0$ (and by Lemma 2.1 , each of $\mathrm{T}(4, \alpha)$ and $\mathrm{T}(5, \alpha)$ is attained at the point $\mathrm{k}=1)$, and for $9 \leq \alpha \leq 15$, each of $\mathrm{T}(2, \alpha), \quad \mathrm{T}(3, \alpha)$ and $\mathrm{T}(4, \alpha)$ is attained at the point $\mathrm{k}=0$ (and by Lemma 2.1 , each of $\mathrm{T}(5, \alpha)$ and $\mathrm{T}(6, \alpha)$ is attained at the point $\mathrm{k}=1$ ).

The lemma below which states that the sequence $\{T(n+1, \alpha)-T(n, \alpha)\}_{n=1}^{\infty}$ isstrictly increasing in $\mathrm{n}$ for any $\alpha(\geq 3)$ fixed is to be proved.

Lemma 2.4 : When $\alpha \neq 2^{i}$ (for any integer $\mathrm{i} \geq 2$ ), $T(n+2, \alpha)-T(n+1, \alpha)>T(n+1, \alpha)-T(n, \alpha)$.

Proof: When $T(n+2, \alpha)=S(n+2,3)$, then $T(n+1, \alpha)=S(n+1,3), T(n, \alpha)=S(n, 3)$, so that $T(n+2, \alpha)-T(n+1, \alpha)=2^{n+1}=2[T(n+1, \alpha)-T(n, \alpha)]$, and so the result holds. Now, let $T(n+2, \alpha) \neq$ $S(n+2,3)$ but $T(n+1, \alpha)=S(n+1,3)$. Let $T(n+2, \alpha)$ be attained at $\mathrm{t}=\mathrm{T}$. Then, since $(n+2, \alpha)=\alpha T(n+2-T, \alpha)+S(T, 3)<S(n+2,3)$,

Then, by Lemma 1.1 and Corollary 1.1,

$T(n+2, \alpha)-T(n+1, \alpha) \geq \alpha[T(n+2-T, \alpha)-$ $T(n+1-T, \alpha)]>\alpha>2^{n}=T(n+1, \alpha)-T(n, \alpha)$.

So, let $T(n+2, \alpha) \neq S(n+2,3)$ and $T(n+1, \alpha) \neq$ $S(n+1,3)$. Let $T(n+2, \alpha), T(n+1, \alpha)$ and $T(n$, $\alpha$ ) be attained at the points $\mathrm{k}=\mathrm{k}_{1}, \mathrm{k}=\mathrm{k}_{2}$ and $\mathrm{k}=\mathrm{k}_{3}$ respectively. Then, by Lemma 1.3 , there are four possibilities that we consider separately below.

Case (A): When $\mathrm{k}_{1}=\mathrm{k}_{2}=\mathrm{k}_{3}=\mathrm{K}$, say. In this case,

$T(n+2, \alpha)-T(n+1, \alpha)=2^{n-K+1}=2[T(n+1, \alpha)-T(n, \alpha)]$.

Case (B): When $\mathrm{k}_{1}=\mathrm{k}_{2}=\mathrm{K}, \mathrm{k}_{3}=\mathrm{K}+1$. Here,

$T(n+2, \alpha)-T(n+1, \alpha)>2^{n-K}=T(n+1, \alpha)-T(n, \alpha)$.

Case $(\mathbf{C})$ : When $\mathrm{k}_{1}=\mathrm{K}, \mathrm{k}_{2}=\mathrm{k}_{3}=\mathrm{K}+1$. In this case,

$T(n+2, \alpha)-T(n+1, \alpha)=2^{n-K}>T(n+1, \alpha)-T(n, \alpha)$.

The result is true in any of the above three cases.

Case (D): When $\mathrm{k}_{1}=\mathrm{K}=\mathrm{K}_{11}$, say, $\mathrm{k}_{2}=\mathrm{K}_{11}+1$, $\mathrm{k}_{3}=\mathrm{K}_{11}+2$. Here,

$T(n+2, \alpha)-T(n+1 \alpha)=\alpha\left[T\left(K_{l l}+2, \alpha\right)-T\left(K_{l 1}+1, \alpha\right)\right]$, $T(n+1, \alpha)-T(n, \alpha)=\alpha\left[T\left(K_{11}+1, \alpha\right)-T\left(K_{11}, \alpha\right)\right]$.

Let $T\left(K_{11}, \alpha\right), T\left(K_{11}+1, \alpha\right)$ and $T\left(K_{11}+2, \alpha\right)$ be attained at $\mathrm{k}=\mathrm{K}_{21}, \mathrm{k}=\mathrm{K}_{22}$ and $\mathrm{k}=\mathrm{K}_{23}$ respectively. The result is true if $\mathrm{K}_{21}, \mathrm{~K}_{22}$ and $\mathrm{K}_{23}$ satisfy one of the three conditions of Case (A), Case (B) and Case (C). In Case (D), we get

$T(n+2, \alpha)-T(n+1, \alpha)=\alpha^{2}\left[T\left(K_{21}+2, \alpha\right)-T\left(K_{21}+1, \alpha\right)\right]$,

$T(n+1, \alpha)-T(n, \alpha)=\alpha^{2}\left[T\left(K_{21}+1, \alpha\right)-T\left(K_{21}, \alpha\right)\right]$.

If $T\left(K_{m l}+2, \alpha\right), T\left(K_{m l}+1, \alpha\right)$ and $T\left(K_{m l}, \alpha\right)$ are attained at the values of $\mathrm{k}$ satisfying one of Case (A), Case (B) and Case (C), then the proof is complete. Otherwise, Case (D) occurs, and need for continuation. Continuing the argument whenever Case (D) occurs, it follows:

$T(n+1, \alpha)-T(n, \alpha)=\alpha^{m}\left[T\left(K_{m l}+1, \alpha\right)-T\left(K_{m l}, \alpha\right)\right]$,

for some integer $m \geq 2$. The analyses of Lemma 2.2 and Lemma 2.3 show that, ultimately, a situation is reached where $T\left(K_{m l}, \alpha\right), T\left(K_{m l}+1\right.$, $\alpha)$ and $T\left(K_{m l}+2, \alpha\right)$ are attained at the values of $\mathrm{k}$ satisfying one of the three cases, namely, Case (A), Case (B) and Case (C). In such a case, the result clearly holds true. 
Lemma 2.4 proves that, for $\alpha \geq 3$ not of the form $2^{\mathrm{i}}(\mathrm{i} \geq 2), T(n+1, \alpha)-T(n, \alpha)$ is strictly increasing in $n$. However, the situation is different if $\alpha=2^{i}$ for any integer $\mathrm{i} \geq 1$. For example,

$M(2)-M(1)=2=M(3)-M(2)$,

$T(3,3)-T(2,3)=4=T(4,3)-T(3,3)$.

Lemma 2.5 : Let $\alpha \neq 2^{i}$ (for any integer $\mathrm{i} \geq 2$ ). Then, there is an infinite number of integers $n \geq$ 1 such that $T(n+1, \alpha)-T(n, \alpha)$ is of the form $2^{\mathrm{s}}$ for some integer $\mathrm{s} \geq 1$.

Proof : Clearly, such an $n$ exists for any $\alpha \geq 3$ (see Corollary 2.1 - Corollary 2.5 in Majumdar (2016) . Now, let $\mathrm{T}(\mathrm{N}-1, \alpha)$ and $\mathrm{T}(\mathrm{N}, \alpha)$ both be attained at $\mathrm{k}=\mathrm{K}$, so that

$T(N, \alpha)-T(N-1, \alpha)=2^{N-K-1}$.

By Lemma 1.5, together with Lemma 1.3, $T(N+$ $1, \alpha)$ is attained at the point $k=K+1$. Let, for some integer $\mathrm{m}>1, T(N+1, \alpha), T(N+2, \alpha), \ldots$, $T(N+m-1, \alpha)$ be attained at the distinct points $\mathrm{k}=\mathrm{K}+1, \mathrm{~K}+2, \ldots, \mathrm{K}+\mathrm{m}-1$ respectively. Then, for $\ell=0,1, \ldots, \mathrm{m}-1$,

$T(N+\ell, \alpha)=\alpha T(K+\ell, \alpha)+S(N-K, 3)$

$<\alpha T(K+\ell-1, \alpha)+S(N-K+1,3)$.

Therefore,

$T(N+\ell, \alpha)-T(N+\ell-1, \alpha)=\alpha[T(K+\ell, \alpha)-T(K$

$+\ell-1, \alpha)] ; \ell=1,2, \ldots, m-1$,

and

$2^{N-K-1}<\alpha[T(K+\ell-1, \alpha)-T(K+\ell-2, \alpha)]<2^{N}$

${ }^{-K} ; \ell=1,2, \ldots, m$.

Now, since the sequence of numbers $\left\{T(K+l-1, \alpha)-T(K+l-2, \alpha\}_{l=100}\right.$ is strictly increasing in $\ell$ (by Lemma 2.4 ), there is an integer $\mathrm{m} \geq 2$ such that

$\alpha[T(K+m, \alpha)-T(K+m-1, \alpha)] \geq 2^{N-K}$.

For the minimum such $\mathrm{m}$, if $\mathrm{m}=\mathrm{M}, T(N+M, \alpha)$ is not attained at the point $\mathrm{k}=\mathrm{K}+\mathrm{M}$, for otherwise,

$T(N+M, \quad \alpha)=\alpha T(K+M, \alpha)+S(N-K, 3)$

$\alpha T(K+M-1, \alpha)+S(N-K+1,3)$, so that

$\alpha[T(K+M, \alpha)-T(K+M-1, \alpha)]<2^{N-K}$, which violates (3). Therefore, $T(N+M, \alpha)$ is attained at $k=K+M-1$ (by Lemma 1.3) with $T(N+M, \alpha)-T(N+M-1, \alpha)=2^{N-K}$.

Corollary 2.2 : Let $\alpha \neq 2^{i}$ (for any integer $\mathrm{i} \geq 2$ ). Let $T(N-1, \alpha)$ and $T(N, \alpha)$ both be attained at the point $\mathrm{k}=\mathrm{K}$, so that

$T(N, \alpha)-T(N-1, \alpha)=2^{N-K-1}$.

Let $\mathrm{M}$ and $\mathrm{L}$ be such that

$T(N+M, \alpha)-T(N+M-1, \alpha)=2^{N-K}, T(L, \alpha)-$ $T(L-1, \alpha)=2^{N-K}-2$.

Then,

(a) $T(N+m, \alpha)$ is attained at the point $\mathrm{k}=\mathrm{K}+\mathrm{m}$ for all $\mathrm{m}=0,1, \ldots, \mathrm{M}-1$,

(b) $T(N+M, \alpha)$ is attained at the point $\mathrm{k}=\mathrm{K}+\mathrm{M}-1$,

(c) $(N+m, \alpha)-T(N+m-1, \alpha)$ is divisible by $\alpha$ for all $\mathrm{m}=1,2, \ldots, \mathrm{M}-1$,

Proof : is evident from the proof of Lemma 2.5.

Since for $\alpha=3$,

$\alpha[T(N, \alpha)-T(N-1, \alpha)]>2^{N}-{ }^{K}>\alpha[T(K+M-$ $1, \alpha)-T(K+M-2, \alpha)]$, and

$\alpha[T(L, \alpha)-T(L-1, \alpha)]<2^{N} \quad-\quad K<\alpha[T(K+M$,

$\alpha)-T(K+M-1, \alpha)]$,

it follows that $\mathrm{N}>\mathrm{K}+\mathrm{M}-1, \mathrm{~K}+\mathrm{M}>\mathrm{L}$. On the other hand, if $\alpha \geq 5, K+M \leq L$, for otherwise,

$T(K+M-1, \alpha)-T(K+M-2, \alpha)>T(L, \alpha)-T(L$ $-1, \alpha)=2^{N-K-2}$,

which gives

$\alpha[T(K+M-1, \alpha)-T(K+M-2, \alpha)]>2^{N-K}$.

But the above result violates the condition (2).

\section{MAIN RESULTS}

In this section, an expression of $T(n, \alpha)$ when $\alpha$ $\neq 2^{i}$ for any integer $i \geq 2$ is presented.

For any $\alpha \geq 3$, let

$a_{n}=T(n, \alpha)-T(n-1, \alpha), n \geq 1$.

Clearly,

$\mathrm{a}_{1}=1, a_{2}=2$ for all $\alpha \geq 3$. 
Now the statement can be made and the following lemma can be proved.

Lemma 3.1 : For all $\mathrm{n} \geq 1, a_{n}$ is of the form $2^{m} \alpha^{\ell}$ for some integers $m \geq 0, \ell \geq 0$.

Proof : is by induction on. Cleary, the result is true when $n=1$ and $n=2$. So, it can be assumed that the result is true for all $\mathrm{i} \leq \mathrm{n}$.

To prove the result for $n+1$, by Lemma 1.3 , the two possibilities that may occur can be considered: If $T(n, \alpha)$ and $T(n+1, \alpha)$ both are attained at $\mathrm{k}=\mathrm{K}$, then

$a_{n+l}=2^{N-K}$;

on the other hand, if $T(n, \alpha)$ is attained at $\mathrm{k}=\mathrm{K}$ and $T(n+1, \alpha)$ is attained at $\mathrm{k}=\mathrm{K}+1$, then

$$
a_{n+1}=\alpha[T(K+1, \alpha)-T(K, \alpha)],
$$

so that, by the induction hypothesis, $a_{n+1}$ is of the form $2^{m} \alpha^{\ell}$.

Lemma 3.2 : Let $\alpha \neq 2^{i}$ for any integer $\mathrm{i} \geq 2$. For $\mathrm{j}$ $\geq 0$, let $\mathrm{k}_{\mathrm{j}}$ be such that $a_{k_{j}}=2^{j}$.

Then, the following results hold.

(a) When $k_{j} \leq n \leq k_{j}+1-1, T(n, \alpha)$ is attained at the point $k=n-j-1$,

(b) $T\left(k_{j+1}, \alpha\right)$ is attained at the point $k=k_{j+1}-j-2$.

Proof: The proof is by induction on $\mathrm{j}$. Since

$\mathrm{k}_{0}=1, \mathrm{k}_{1}=2$,

and since $T\left(k_{0}, \alpha\right)=T(1, \alpha)$ is attained at the point $\mathrm{k}=0$, and $T\left(k_{1}, \alpha\right)=T(2, \alpha)$ is attained at $\mathrm{k}=0$, the result is true for $\mathrm{j}=0$. So, assume it can be that the result is true for some $\mathrm{j}$. Then, in the notation of Corollary 2.2,

$N=k_{j}, N-K-1=j, N+M=k_{j+1}$.

(a) From part (a) of Corollary 2.2, for all $\mathrm{m}$ satisfying $k_{j} \leq N+m \leq k_{j+1}-1, T(N+m, \alpha)$ is attained at $k=K+m=N+m-j-1$.

(b) By part (b) of Corollary 2.2, $T\left(k_{j+1}, \alpha\right)$ is attained at $k=K+M-1=k_{j+1}-j-2$.
Thus, the result is true for $\mathrm{j}+1$, completing induction.

Corollary 3.1 : Let $\alpha \neq 2^{\mathrm{i}}$ for any integer $\mathrm{i} \geq 2$. For $\mathrm{j} \geq 0$, let $\mathrm{k}_{\mathrm{j}}$ be such that $a_{k_{j}}=2^{j}$. Then, for m with $1 \leq m \leq k_{j+1}-k_{j}-1$,

$T\left(k_{j}+m, \alpha\right)-T\left(k_{j}+m-1, \alpha\right)=\alpha\left[T\left(k_{j}+m-j-1\right.\right.$, a) $\left.-T\left(k_{j}+m-j-2, \alpha\right)\right]$.

Proof : follows from the proof of part (a) of Lemma 3.2, using the fact that $T\left(k_{j}+m, \alpha\right)$ is attained at $k=k_{j}+m-j-1$, and $\left.T\left(k_{j}+m-1, \alpha\right)\right]$ is attained at $k=k_{j}+m-j-2$. Let $\left\{b_{n}\right\}_{n=1}^{\infty}$ be the sequence of numbers, defined as follows : $b_{n}=2^{m} \alpha^{\ell} ; m, \ell \geq 0$,

arranged in ascending order, where $\alpha \geq 3$ is an integer. Clearly,

$b_{1}=1, b_{2}=2$.

It is noted that the sequence $\left\{b_{n}\right\}_{n=1}^{\infty}$ is strictly increasing if $\alpha \neq 2^{i}$ (for any integer $i \geq 2$ ).

When $\alpha=3$, the first few terms of the sequence $\left\{b_{n}\right\}_{n=1}^{\infty}$ are

1, 2, 3, 4, 6,8,9,12,16,18,

when $\alpha=5$, the first few terms are

$1,2,4,5,8,10,16,20,25,32$,

and when $\alpha=6$, the first few terms are

$1,2,4,6,8,12,16,24,32,36$,

The sequence $\left\{b_{n}\right\}_{n=1}^{\infty}$ has been studied by Matsuura et al. (2008) to some extent, who gives a recurrence relation satisfied by the terms of the sequence $\left\{b_{n}\right\}_{n=1}^{\infty}$.

Given any $\alpha \neq 2^{i}$ for any integer $i \geq 2$, and any integer $\mathrm{j} \geq 0$, a related problem of interest is to find the number of elements of the sequence $\left\{b_{n}\right\}_{n=1}^{\infty}$ such that

$\alpha^{\mathrm{j}}<2^{\mathrm{m}} \quad \alpha^{\ell}<\alpha^{\mathrm{j}+1}$

Now, since (3.6) is equivalent to

$\alpha^{j-\ell}<2^{m}<\alpha^{j-\ell+1}$, we see that m must satisfy the following inequality:

$(j-\ell) \frac{\ln \alpha}{\ln 2}<m<(j-\ell+1) \frac{\ln \alpha}{\ln 2}$ 
Let $N_{\alpha}(j)$ denote the number of elements of the sequence $\left\{b_{n}\right\}_{n=1}^{\infty}$ satisfying the inequality (3.6), that is, let

$N_{\alpha}(j)=\left|\left\{b_{n}: \alpha^{j}<b_{n}<\alpha^{j+1}\right\}\right|=\left|\left\{b_{n}: \alpha^{j}<2^{m} \alpha^{l}<\alpha^{j+1}\right\}\right|$

Then, the following lemma, giving the recurrence relation satisfied by $N_{\alpha}(j)$ is obtained.

Lemma 3.3 : For any integer $\mathrm{j} \geq 0$,

$$
N_{\alpha}(j+1)=N_{\alpha}(j)+\left|\left\{m: \alpha^{j+1}<2^{m}<\alpha^{j+2}\right\}\right|
$$

Proof : The second term on the right-hand side above corresponds to the case with $\mathrm{j}+1$ and $\ell=$ 0 in (3.7); also, from (3.7), the case for $j+1$ with $\ell=1$ corresponds to the case $\mathrm{j}$ with $\ell=0$, the case for $\mathrm{j}+1$ with $\ell=2$ corresponds to the case $\mathrm{j}$ with $\ell=1$, and so on. Hence, the result follows.

Lemma 3.4 : For any integer $\mathrm{j} \geq 0$,

$N_{\alpha}(j) \equiv\left|\left\{b_{n}: \alpha^{j}<b_{n}<\alpha^{j+1}\right\}\right|=\max \left\{m: 2^{m}<\alpha^{j+1}\right\}$.

Proof : Let $2^{\mathrm{i}}<\alpha<2^{\mathrm{i}+1}$ for some integer $\mathrm{i} \geq 1$. Then, when $\mathrm{j}=0, \mathrm{~m}=\mathrm{i}$. Thus, the result is true for $\mathrm{j}=0$. To proceed by induction on $\mathrm{j}$, we assume that the result is true for $\mathrm{j}$. Then, the validity of the result for $\mathrm{j}+1$ follows from Lemma 3.3.

Corollary 3.2 : For any integer $\mathrm{j} \geq 1$,

$N_{\alpha}(j) \equiv\left|\left\{b_{n}: \alpha^{j}<b_{n}<\alpha^{j+1}\right\}\right|=\left\lfloor(j+1) \frac{\ln \alpha}{\ln 2}\right\rfloor$, where $\lfloor x\rfloor$ is the floor of the real number $x>0$.

Proof : is immediate from Lemma 3.4.

The following result, due to Matsuura et al. (2008), gives the recurrence relation satisfied by the sequence $\left\{b_{n}\right\}_{n=1}^{\infty}$.

Lemma 3.5 : Let $\alpha \neq 2^{i}$ for any integer $i \geq 2$. Then, for any n with $2^{j}<b_{n}<2^{j+1}$,

$b_{n}=\alpha b_{n-(j+1)}$.
The result below has been established (Matsuura at al. 2008), who used induction on $\mathrm{n}$ to prove it, making use of the properties of the sequence $\left\{b_{n}\right\}_{n=1}^{\infty}$. An alternative proof can be proveded.

Theorem 3.1 : For all $\mathrm{n} \geq 1, a_{n}=b_{n}$.

Proof : When $\alpha \neq 2^{\mathrm{i}}$ for any integer $\mathrm{i} \geq 2$, by Corollary 3.2, for n satisfying $k_{j}<n<k_{j}+1$,

$a_{k_{j}+m}=\alpha a_{k_{j}+m-j-1} ; 1 \leq m \leq k_{j+1}-k_{j}-1$.

This, together with Lemma 3.5, shows that the sequences $\left\{b_{n}\right\}_{n=1}^{\infty}$ and $\left\{a_{n}\right\}_{n=1}^{\infty}$ satisfy the same recurrence formulas. Hence, $a_{n}=b_{n}$.

By virtue of Theorem 3.1, it can be expressed as the solution of the recurrence relation (1.2) in terms of the sequence $\left\{b_{n}\right\}_{n=1}^{\infty}$. This is as below.

Theorem 3.2 : Let $\alpha \neq 2^{i}$ for any integer $\mathrm{i} \geq 2$.

Then,

$T(n, \alpha)=\sum_{j=1}^{n} b_{j}$.

Moreover, for any integer $\mathrm{j} \geq 0, T\left(k_{j}, \alpha\right)$ is attained at the point $k=k_{j}-j-1$, and for $\mathrm{n}$ satisfying $k_{j} \leq n \leq k_{j+1}-1, T(n, \alpha)$ is attained at the point $k=n-j-1$, where $\mathrm{k}_{\mathrm{j}}$ is given by (3.2).

Proof : Writing $T(n, \alpha)$ as follows :

$T(n, \alpha)=\sum_{j=1}^{n}[T(j, \alpha)-T(j-1, \alpha)]$,

and then making use of Theorem 3.1, the desired expression for $T(n, \alpha)$ is obtained. The remaining part follows from Lemma 3.2.

\section{DISCUSSION}

This paper derives some new results in connection with the difference $T(n+1, \alpha)-T(n, \alpha)$, which plays a vital role in solving the recurrence relation (1.2). 
These results help understand the combinatorial properties inherent in the function $T(n, \alpha)$, when $\alpha \neq 2^{i}$ for any integer $i \geq 2$. For example, results similar to those in Lemma 3.2 appears in Matsuura et al. (2008) as well, but the proof there is by induction, making use of the properties of the sequence $\left\{b_{n}\right\}_{n=1}^{\infty}$, while the present analysis shows that they can, in fact, be derived independently from the properties of the function $T(n, \alpha)$ alone. Again, the result given in Lemma 3.4 has been established by Matsuura $e t$ al. (2008), but a simplified proof through Lemma 3.3, is now obtained which gives the recurrence relation satisfied by $N_{\alpha}(j)$.

From Theorem 3.2, it is seen that, in order to find $T(n, \alpha)$, one has to keep track of the sequence $\left\{b_{n}\right\}_{n=1}^{\infty}$. For small values of n, $T(n$, $\alpha)$ can be calculated. For example, adding the first eight terms of the sequence of numbers in (3.3), $\mathrm{T}(8,3)=45$ is obtained. Again, adding the first six terms of the sequence of numbers in (3.4), $\mathrm{T}(6,5)=30$, and adding the first five terms of the sequence of numbers in (3.5), $\mathrm{T}(5,6)=21$ are obtained. However, for large values of $\mathrm{n}$, to find $T(n, \alpha)$ (for any $\alpha \geq 3$ fixed) one has to find $b_{n}$ using the recurrence relation given in Lemma 3.5. To do so, the value of $k_{j}=k_{j}(\alpha)$, given by (3.2) need to be considered. Thus, for example, to find $T(14,3)$, all the values from $b_{1}$ through $b_{14}$ (corresponding to $\alpha=3$ ) to be considered. To find $b_{14}$ using Lemma 3.5 , one has find $\mathrm{j}$ such that $2^{\mathrm{j}}<\mathrm{b}_{14}<2^{\mathrm{j}+1}$. To know $\mathrm{j}$ (and $\mathrm{k}_{\mathrm{j}}$ ) such that $a_{k_{j}}=2^{j}$. But in the current literature, no such result is available. Therefore, from the computational point of view, Theorem 3.2 is not of much use. However, Lemma 1.3 can be exploited to find $T(n, \alpha)$ as well as the value of $\mathrm{k}$ where $T(n, \alpha)$ is attained, recursively in $\mathrm{n}$. Thus, assuming that $T(n, \alpha)$ is attained at $\mathrm{k}=\mathrm{K}$, the problem of finding $T(n+1, \alpha)$ reduces to $T(n+1$, $\alpha)=\min \{\alpha T(K, \alpha)+S(n-K+1,3), \alpha T(K+1$, $\alpha)+S(n-K, 3)\}$.

Using the above algorithm, the values of $T(n, \alpha)$ may be calculated recursively in $n$. One such recursive algorithm is presented by Ziauddin and Majumdar et al. (2017).

When $\alpha=2$, exactly one of the following two alternatives holds true :

Case (1): $T(n+2, \alpha)-T(n+1, \alpha)=2[T(n+1$, $\alpha)-T(n, \alpha)]$,

Case (2) : $T(n+2, \alpha)-T(n+1, \alpha)=T(n+1, \alpha)$ $-T(n, \alpha)$.

When $\alpha \neq 2^{i}$ for any integer $i \geq 2$, by Lemma 2.4 , it is seen that the relationship (5) cannot hold true. The relationship (4) clearly holds true when $T(n+$ $2, \alpha)=S(n+2,3)$. To see whether (4) holds in other cases as well, it is necessary to study in more detail the values of $\mathrm{k}$ at which $T(n+2, \alpha), T(n+1, \alpha)$ and $T(n, \alpha)$ are attained. In this context, the following two results can be proved.

Lemma 4.1 : Let $2^{i}<\alpha<2^{i+1}$ for some integer i $\geq 1$. Let $T(n, \alpha)$ be attained at the point $\mathrm{k}=0$. Then, $T(n+2, \alpha)$ is not attained at $\mathrm{k}=2$.

Proof : Let $T(n, \alpha)$ be attained at $\mathrm{k}=0$, so that, by Corollary 1.1, $\alpha>2^{n-1}$. Now, if $T(n+2, \alpha)$ is attained at $\mathrm{k}=2$, then

$T(n+2, \alpha)=\alpha T(2 \alpha)+S(n, 3)<\alpha T(1, \alpha)+S(n+1,3)$,

giving $\alpha<2^{n-1}$, and to a contradiction is reasched.

Lemma 4.2 : Let $2^{i}<\alpha<2^{i+1}$ for some integer $\mathrm{i} \geq 1$. Let $T(n, \alpha)$ be attained at the point $\mathrm{k}=1$. Then, $T(n+2, \alpha)$ is not attained at $\mathrm{k}=3$.

Proof : Let $T(n, \alpha)$ be attained at $\mathrm{k}=1$, so that,

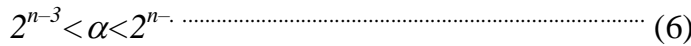
(see the proof of Lemma 2.1). Then, $T(n+2, \alpha)$ is not attained at $\mathrm{k}=3$, for otherwise 
$T(n+2, \alpha)=\alpha T(3, \alpha)+S(n-1,3)<\alpha T(2, \alpha)$

$+S(n, 3)$,

which gives $\alpha<2^{n-3}$, if $\alpha \geq 5$. And a contradiction is reached. If $\alpha=3$, from (7) it is, $9<2^{n-1}$.

On the other hand, corresponding to $\alpha=3$, the only values of $n$ satisfying (6) are $n=3,4$, and in either case, (8) is violated.

A close analysis shows that, there are four possibilities such that $T\left(K_{m 1}, \alpha\right), T\left(K_{m 2}+1, \alpha\right)$ and $T\left(K_{m l}+2, \alpha\right)$ (in the proof of Lemma 2.4) are attained :

(1) $T\left(K_{m 1}, \alpha\right)$ and $T\left(K_{m 2}+1, \alpha\right)$ are attained at $\mathrm{k}$ $=0, T\left(K_{m 2}+2, \alpha\right)$ is attained at $\mathrm{k}=1$,

(2) $T\left(K_{m 1}, \alpha\right)$ is attained at $\mathrm{k}=0, T\left(K_{m 2}+1, \alpha\right)$ and $T\left(K_{m 2}+2, \alpha\right)$ are attained at $\mathrm{k}=1$,

(3) $T\left(K_{m 1}, \alpha\right)$ and $\mathrm{T}\left(\mathrm{K}_{\mathrm{m} 2}+1, \alpha\right)$ are attained at $\mathrm{k}$ $=1, T\left(K_{m 2}+2, \alpha\right)$ is attained at $\mathrm{k}=2$,

(4) $\mathrm{T}\left(\mathrm{K}_{\mathrm{m} 1}, \alpha\right)$ is attained at $\mathrm{k}=1, T\left(K_{m 2}+1, \alpha\right)$ and $T\left(K_{m 2}+2, \alpha\right)$ are attained at $\mathrm{k}=2$.

In any of the above four cases, the relationship (4) does not hold. This observation leads us to conjecture that (4) holds only when $T(n+2, \alpha)=$ $S(n+2,3)$.

\section{ACKNOWLEDGEMENT}

Part of the materials given here were carried out during the Academic Development Leave (ADL) during April - September, 2018. The author wishes to acknowledge with thanks, Ritsumeikan Asia-Pacific University, Japan, for granting the ADL.

\section{REFERENCES}

Hinz, A.M. An Iterative Algorithm for the Tower of Hanoi with Four Pegs. Computing, 1989; 42: 133 - 140.

Majumdar, A.A.K. Some Local-Value Relationships for the Recurrence Relation Related to the Tower of Hanoi Problem, Proceedings of the Pakistan Academy of Sciences, A: Physical and Computational Sciences, 2016; 53(2): 187 - 201.

Majumdar, A.A.K. The Classical Tower of Hanoi Problem and Its Generalizations, Vol. 1 : Multi-Peg Generalization. Lambert Academic Publishing, U.S.A. 2012.

Majumdar, A.A.K. The Generalized Four-Peg Tower of Hanoi Problem. Optimization, 1994; 29: 349-360.

Matsuura, A. Exact Analysis of the Recurrence Relations Generalized from the Tower of Hanoi. SIAM Proceedings in Applied Mathematics, 2008; 129: pp. 228 - 233.

Roth, T. The Tower of Brahma Revisited. J. Recreational Mathematics, 1974; 7(2): 116 - 119.

Stockmeyer, Paul K. Variations of the Four-Post Tower of Hanoi Puzzle. Congressus Numerantium, 1994; 102: 3 - 12.

Ziauddin Ahmed, A.K. and Majumdar, A.A.K. On a Recurrence Relation Related to the Reve's Puzzle : Computational Aspect. Jahangirnagar Journal of Mathematics \& Mathematical Sciences, 2017; 30: $105-111$

(Received revised manuscript on 12 July 2018) 